\title{
Local Helioseismology - What Does It Really Tell Us?
}

\author{
Frank Hill \\ National Solar Observatory, PO Box 26732, Tucson Arizona USA \\ 85726-6732
}

\begin{abstract}
The three major local helioseismology methods of time-distance, ring diagrams, and acoustic holography are briefly summarized along with their advantages and disadvantages. Useful tests with artificial data are outlined, and current data comparisons briefly described.
\end{abstract}

\section{Introduction}

The subfield of local helioseismology is now 15 years old. In its brief history, it has yielded maps of flows beneath the solar surface, images of active regions on the far-side of the Sun, and inferences about the sound speed below sunspots. However, there has not yet been a systematic study of the consistency among results obtained with the three major local methods of time-distance, acoustic holography, and ring diagrams. In addition, realistic numerical simulations of data suitable for testing the methods have proven difficult to construct. This paper gives a brief overview of the methods, comparing them from a heuristic point of view. It also discusses the types of tests that would be desirable, and presents the latest results from a data-based comparison exercise now underway.

\section{Ring Diagrams}

Ring diagrams (Hill 1988) are constructed by computing the three-dimensional power spectrum in a local area on the solar surface. Slicing the spectrum at constant temporal frequency $\nu$ reveals the solar oscillation signal as a set of concentric rings. The offset of the rings in horizontal wavenumber components $k_{x}$ and $k_{y}$ is a measure of the subsurface flow. The analysis is typically done with a "dense-pack" of 189 areas of diameter $15^{\circ}$ and spacing of $7.5^{\circ}$ in heliographic coordinates. The ring offset is then inverted to infer the flows as a function of depth below each area.

The advantages of the method are that it is straightforward; the effective averaging over the area reduces the random errors; the inversion is easy to perform; and the resolution kernels of the inversion are simple to interpret. The disadvantages are the relatively low spatial resolution; it is mainly limited to near-surface layers; and the results may be sensitive to the absorption of $p$ modes by sunspots. 


\section{Time-Distance}

The time-distance method (Duvall et al. 1993) is very similar to the methods of terrestrial seismology. An acoustic source emits sound which travels along the ray paths prescribed by the solar structure and the properties of the acoustic emission. The observed relationship between the distance from the source and the acoustic travel time is a measure of conditions below the surface. The waves can be reflected multiple times within their thermal cavity. Time-distance diagrams are constructed either from the $\ell-\nu$ diagram, or from velocity crosscorrelations. A typical analysis period is 8 hours.

The one major advantage of time-distance is the high spatial resolution of less than 1 arc min that can be achieved with the method. There are a number of disadvantages - the inversion is difficult; the averaging kernels are hard to interpret; it is limited primarily to near-surface layers; the errors in the results cannot be simply estimated; and the height of formation of the spectral line used for the observations can introduce additional time delays in localized areas.

\section{Holography}

Acoustic holography (Lindsey \& Braun 1990) is completely analogous to optical holography. The method analyzes the pattern of interference of acoustic waves that are either emitted from sources or are scattered from subsurface inhomogeneities. The observed interference pattern is computationally regressed through a solar model to infer the structure below the surface. The method can control the depth of the focal plane to bring structures into view.

The advantages of the method are the high spatial resolution that can be achieved; the ability to infer the local sound speed; and the ability to probe very deeply. Indeed, holographic images of the far side of the Sun are now routinely made and have proven valuable for solar activity and space weather forecasting. The disadvantages are the difficulty of removing the influence of the surface on the results; the absence of averaging kernels and error estimates; and the difficulty of inferring velocity fields.

\section{General Limits}

All three methods have four common limitations. Perhaps the most worrisome is that the basic assumption of the response of the wave field to the flows is theoretically and numerically unverified. This shortcoming should be resolved soon as several efforts to simulate the observations are underway. A second disadvantage is that the methods are insensitive to the vertical component of the velocity field. Since the waves must travel both downward and upward, the effects of vertical flows cancel out to first order and only second-order effects survive.

All of the methods are limited by a trade-off between depth and spatial resolution. Since fewer modes reach deeper depths, the best horizontal spatial resolution is achieved near the surface and degrades with depth. Finally, the spherical surface of the Sun causes the horizontal spatial Nyquist wavenumber 
to decrease near the limb. This foreshortening limits the local results to near disk center.

\section{Tests}

In the absence of theoretical and numerical confirmation of the methods, work has shifted to comparisons of methods on common data sets and to comparison of data sets with common methods. The Local Helioseismology Comparison (LoHCo) group has been formed for this purpose. This group has compared ring-diagram flow fields from GONG and MDI data sets; ring-diagram and timedistance flows from a common MDI data set; time-distance travel times from GONG and MDI; and both near- and far-side holography on GONG and MDI. The results can be simply summarized: there is large-scale agreement between methods and data sets, with differences in the details. More specifically, the results concerning rotation and torsional oscillations are robust, but the inferences about counter-cell meridional flows, flows below sunspots, and changes in the tachocline are less so. Not surprisingly, the noise level in the observations plays a role, particularly when it is a function of spherical harmonic degree.

The major drawback of observational comparisons is that the actual structure of the Sun is unknown. Thus, an unsuspected systematic error common to all methods could dominate the results and lead to unjustified complacency. This has motivated a number of efforts to simulate the observations including a known solar internal structure or flow field. The full numerical simulation is quite formidable, as it requires a physically self-consistent wave field and flow field. Several approximations to the problem are currently in progress, such as the construction of the wave field from an artificial ring-diagram spectrum without attempting self-consistency. Even this "physics-lite" approach is valuable, particularly with a zero flow field as a null test.

\section{Conclusion}

Local helioseismic methods can provide images of velocity fields and sound speed structures in the outer solar layers. These images provide important information on the origin of the solar activity cycle and the solar internal dynamics. However, much remains to be done in the testing and verification of these methods.

\section{References}

Duvall, T.L. Jr., Jefferies, S.M., Harvey, J.W., \& Pomerantz, M.A. 1993, Nature, 362, 430

Hill, F. 1988, ApJ, 333, 996

Lindsey, C., and Braun D.C. 1990, Solar Phys, 126, 101 